\title{
EINFÜHRUNG
}

IN DIE

\section{THEORIE DER WÄRME}

\author{
VON
}

\author{
DR. HEINRICH MACHE \\ O. Ö. PROFESSOR AN DER TECHNISCHEN HOCHSCHULE \\ IN WIEN
}

MIT 96 TEXTFIGUREN

BERLIN UND LEIPZIG 1921

VEREINIGUNG WISSENSCHAFTLICHER VERLEGER WALTER DE GRUYTER \& CO.

vORMALS G. J. GOSCHEN'SCHE VERLAGSHANDLUNG :: J. GUTTENTAG, vERLAGSBUCHHANDLUNG :: GEORG REIMER :: KARL J. TRÜBNER :: VEIT \& COMIP. 
Alle Kechte, einschlieBlich des Ubersetzungsrechts, vorbehalten.

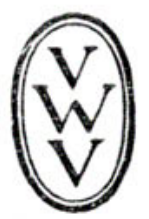

